\title{
Technology Innovation of Product Using CAI System Based on TRIZ
}

\author{
Zhang Jianhui, Yang Bojun, Tian Yumei and Tan Runhua \\ Institute of Design for Innovation, Hebei University of Technology \\ 300130, Tianjin, P.R.China, \\ zjh2031@sina.com \\ home page: http://www.triz.com.cn
}

\begin{abstract}
Technology innovation and creative problem solving are necessary for long-term enterprise survival. Making strategic decisions for product development is a key step in the technical innovation process. Computer-aided innovation (CAI) systems based on TRIZ (Theory of inventive problem solving) support strategic ideas generation of innovation. TRIZ methods are applied to assess the maturity of a technical system and to forecast future technological R\&D plans. A general process for technology innovation of product supported by CAI system is developed. A case study is presented to show how technology maturity mapping and patterns and lines of evolution are used to predict the future development of butterfly valve's sealing technology.
\end{abstract}

\section{Introduction}

Making strategic decisions for product development is one of the toughest jobs that managers of Research and Development have in an organization. Deciding between optimizing existing technologies and developing new core technology is one of them [1]. TRIZ (Theory of inventive problem solving) is emerging as a powerful scientific tool that is applied to assess the maturity of a technical system and helps decision-makers to make strategic forecasting decisions.

Every product is a technical system based on its core technology, and the technical system evolves over time, which process is a macro-level methodology aimed at the maturity of an existing technology. What evolve are characteristic indices of the technical systems and its improvement can be shown by S-curve on the time-scale. The stages of the S-curve are infancy, growth, maturity and decline [2,3]. The position of current product on its evolutionary S-curve is called the technology maturity of product (TM) and the process of identifying TM is called the product technology maturity mapping (TMM) $[1,4]$. 
Understanding the TM allows strategic decisions to be made concerning the optimization or innovation related to product development strategies. Once the TM of product is determined, patterns and lines of technological evolution belong to technological forecasting tools of TRIZ can be used to find some technology opportunities and to forecast future technological R\&D plans. The Computer-aided innovation (CAI) systems based on TRIZ are practical tools to support this process, which can accelerate the technology innovation process of product.

This paper introduces a new method for TMM and give elements to guide the optimization or innovation decision involved in product development strategies. Technology forecast tools of TRIZ are applied to help decision makers to determine the best way forward. A general process for technology innovation of product supported by CAI system is formed.

\section{Technology Maturity Mapping}

Technology advancement is a principal impetus in economic development. Foreseeing technological advancement that will shape the future is of immense importance for many organizations, since they can be deeply influenced by emerging innovations [5]. Assessment of an organization's current technology should drive the direction of the R\&D planning process. Ellen Domb [8] Suggest that "people tend to do an initial assessment of their product maturity base on their emotional state." An assessing technology, based upon a systematic logical analysis, should be introduced.

Altshuller found out that technical system evolution as a function of time was related to four primary S-curve descriptors: the number of inventions in the field, the level of those inventions, the performance of the technology, and the profitability of the technology [2]. Data pertaining to these descriptors can indicate the position of the technology on the main biological S-curve, so the maturity of technical system can be assessment $[1,4]$.

But to some products the performance index and profitability index could not be expressed exactly by a single parameter that can be gotten directly, and sometimes the data could not be gotten [6]. For example, different industries each have different curves for profitability vs. time. Darrell Mann [7] mainly examined the quantity distribution of two kinds of patent, which are number of cost reduction related patents and symptom curing related patents, to refine the maturity of a system. The number of such inventions tends to increase as the system matures.

Integrating the fruits of Altshuller and Darrell Mann on TMM, Zhang [6] uses patent level, patent number and number of symptom curing patent (SCP) as criteria to map the technology maturity of a product. The standard curves are shown in figure 1. Patent number represents the active degree of technical innovations of the product. The patent level reflects the level of innovations. Altshuller divided all patents into five levels to measure the importance of the patents [2,3]. Symptom curing patent means patents which focus on curing problems which emerge as a result of earlier inventions by introducing implement technology, structure or parts rather than search for substitute technology without relative limitation [7]. And the change of the quantity distribution shows the shift of the improvement keystone. 

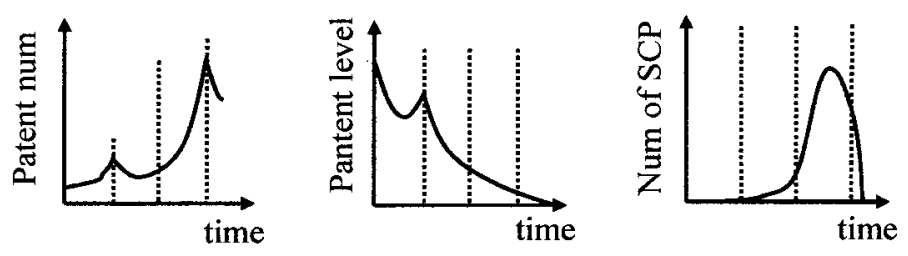

Fig. 1. Product Patent Attribute Curves.

The three curves share common data source, and each shows a patent attribute of product respectively [6]. Each of the Product Patent Attribute Curves (PPAC) can be constructed by collecting patent data that relevant to the system under study. The shapes of each of the curves are compared with the shapes of the classical curves, and then a composite analysis of the three curves reveals a data-driven assessment of the maturity of the technological system.

\section{Optimization or Innovation Dilemma Solving Using CAI}

Once the TM of a company's product has been assessed, the future R\&D direction can be decided by decision-makers. A strategic decision for company is the dilemma of the optimization versus innovation. Should further investments be made to optimize the technology around the core technology? Or should investments be made to develop a new core technology to replace the existing core technology [1]?

To generate strategic ideas successfully and reduce uncertainties effectively are essential to the future R\&D direction. The point of view of this study is that the patterns and lines of evolution belong to technological forecasting tool of TRIZ can be used to generate ideas in a systematic manner with reducing uncertainties. In addition, most of the tools of TRIZ are include in CAI systems. So strategic decision generation supported by CAI systems is possible.

Generally, the patents of the existing product should be gotten first and TMM then should be made. If the core technology of the product under study is in the mature or decline stage, actions of innovation of the core technology should be taken firstly. If the core technology is in the infancy or growth stage, actions of optimization of the core technology should be taken firstly. Once the TM of a product is determined, the patterns and lines of technological evolutions can be applied to forecast future technological development.

The development CAI systems based on TRIZ has made TRIZ more applicable and practical. A few CAI systems, such as Goldfire Innovator of Invention Machine (USA), IWB of Ideation International (USA), InventionTool (China) have been developed and applied by industries. They include data-base for technological evolution, inventive principles, effects, etc. And the InventionTool has the TMM function module. The application of these systems makes the designers to find problem solutions more easily in a short time. 


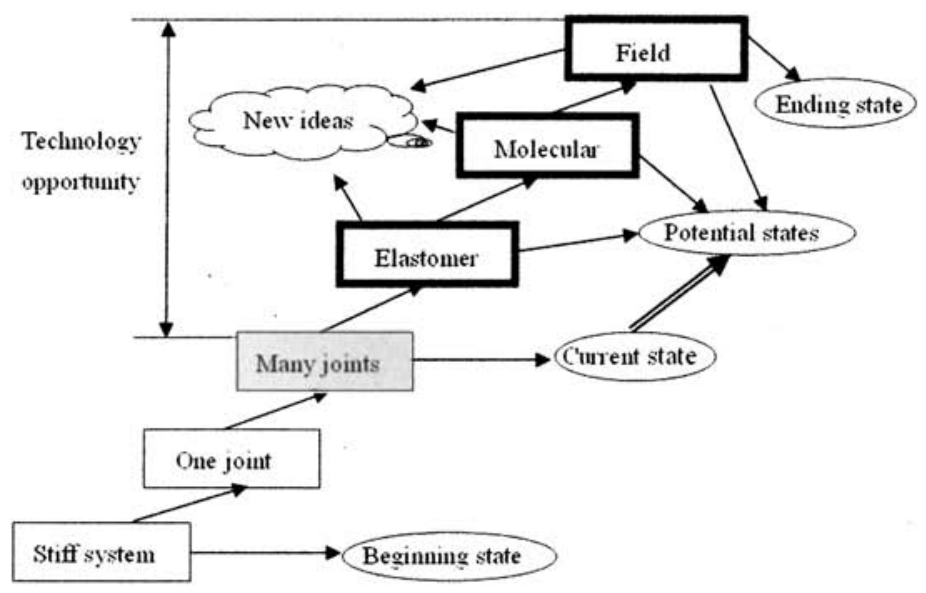

Fig. 2. A line of technological systems evolution

\section{Technology Opportunity Identifying}

Patterns and lines of technological evolution belong to technological forecasting of TRIZ [8], which is a proactive approach to forecasting developed during the $1970 \mathrm{~s}$

and now is also in development. Technological Forecasting reports the probabilities of certain design parameters falling within particular confidence intervals at some future time. Critical design advances for future products and processes can be identified by applying the patterns and lines. With these advances, the field of parameters is narrowed and a tighter range for confidence levels is defined.

The patterns are very helpful for technology forecasting since they identify the most effective directions for the system's development. A pattern of evolution delineates a general direction for further system transformation but says nothing about the details of this transformation. The lines of technological evolution under each pattern describe more specifically the stages of the systems development and therefore provide even greater predicting power.

Figure 2 is a line under pattern: Evolution Toward Increased Dynamism and Controllability. There six states shown in this line which are stiff system, one joint, many joints, elastomer, molecular and field. The beginning state for this line is stiff system and the ending state is field. If the technology of the product under study is in the state of "many joint", the future states of technology evolution may be elastomer, molecular, or field. Every future state may imply one or a few ideas for the future development of the technology. The distance between the ending state and the current state in that line is a technology opportunity or a technology gap. From the line a technology opportunity can be found and several new ideas can also be generated [9]. 
Technology opportunities for the existing product under study can be found by searching different patterns of evolution. First, a pattern is selected and a line under this pattern is also selected. Then, current state for the product is determined from the line selected. By this searching method one or a few technology opportunities can be identified.

Every technology opportunity includes one or several potential states, which are upper states from the current to the ending state. Every potential state implies one or a few new ideas of potential technologies to be used for the evolution of current technology in the future development.

\section{A general process for technology innovation of product supported by CAI system}

The technological systems evolve over time with the functional efficiencies of key characteristics of the technical system increase dramatically. Each function is associated with a certain part of the technical system (product or process), and the various forms of technical innovations indicate the evolution of functions. The key patents about a certain product are the track record of core technical innovation of the product and can represent the history of product evolution. So the patent information of the product can be treated as the main source of product evolution analysis. A general process of new idea generation for technology innovation supported by CAI includes the following six steps:

Step 1: Product patent retrieval and filtration

To an enterprise the first work is to define its local or international competitor, to select patent databases in competitive range, and to use the key word to retrieve all patent relevant to the product. In addition, those irrelevant patents to the object evolution in nature should be excluded. For example, the patent about product's appearance is irrelevant to the technological evolution of product, and can be excluded.

Step 2: Analyze the history of product evolution and assess its TM

The analysis of key patent information can provide with valuable technical and business information regarding target or key technology of the product. At the same time, patent level, patent number and number of SCP can be available, so the PPAC can be constructed and the TM of the product can be mapped. InventionTool3.0 (China), which is a CAI system, supports this step.

Step 3: Decision between optimization and innovation

If the core technology of the product under study is in the mature or decline stage, innovation of the core technology is preferred. If the core technology is in the infancy or growth stage, optimization of the core technology is preferred.

Step 4: Select relevant patterns and lines of evolution in CAI systems to analyze the product and make strategic decision

Apply the patterns and lines that matched the evolution of product under study to analyze the product and making a connection. So the current state and potential states can be determined, and the technology opportunity can be identified to forecast future technological development. 
Most of the tools of TRIZ are include in CAI systems. And the CAI systems, such as Goldfire Innovator of Invention Machine (USA) and InventionTool 3.0 (China) can support this step.

Step 5: Generate strategic new ideas

Under analyzing the technology opportunity that founded in the relevant line, some strategic new ideas for future R\&D direction of product can be generated.

Step 6: Evaluate new ideas

Decide if it is feasible to support possible future goals using the new ideas, and if it is, then select the development to be implemented, otherwise, ignore the ideas.

\section{Case Study}

Butterfly valve is a controlling valve used in large-size pipe, which has function of opening or closing pipe, regulating flux and check. It initially comes from the baffle of chimney or flue that regulate the scavenge capacity. And today it has been used abroad in chemicals, metallurgy, waste treatment, and so on. A butterfly valve has several characteristic indices such as integral leakage, maximum working press, times of open and close, maximum rotational moment. And yet one index improvement can make another decline. Then finding an integral characteristic index is not duck soap.

Step 1: Product patent retrieval and filtration

It is difficult to know when the first butterfly was designed and developed in China. The key word "butterfly valve" is used to retrieve relevant patents from Chinese patent base. 688 patents are retrieved and some of them were eliminated and at last about 600 patents from 1985 to 2005 are selected as resources to carry out this study.

Step 2: Analyze the history of product evolution and assess its TM

According to study tends of the technology evolution for the main structure of the butterfly valves, it is divided into main three modules: the controlling module (includes drive and transmission section), executing module (as shown in figure 3 , includes valve disc, valve shaft, valve seat, and seal ring) and valve body. The evolution of the executing module shows the core technology changes of the seal structure of this kind of valve.

The CAI system, InventionTool3.0 is applied to carry out the TMM. After determining the level, totaling year after year, proportion of SCP, these information are input into the TMM functional module of InventionTool. Then conic and cubic are selected to fit curves, which are shown in figure 4 with the classical curves. In the last five years the proportion of SCP to total was about $70 \%$. By analysis of the Product Patent Attribute Curves in figure 4, a conclusion is made that the butterfly valve of the country is in its maturity stage.

Step 3: Decision between optimization and innovation

By the conclusion in step 2, the butterfly valve technology can look forward to a new core technology. Though, optimization of the auxiliary, secondary or harmful functions can keep on gain profit but it will not allow the valve firm to stay competitive for the long run. Decision should be make now to insure a long-term profit. In recently years, much demand has arisen for new substitute technology and 
it is likely that this trend will continue. So, further investments should be made to develop the new technology around the core technology.

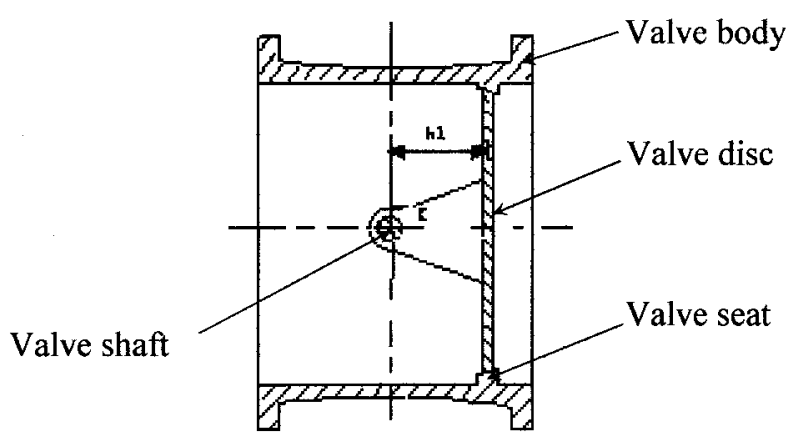

Fig. 3. The main structure of butterfly valve

Step 4: Select relevant patterns and lines of evolution in CAI systems to analyze the product and make strategic decision

On the basis of the above analysis, patterns and lines of evolution should be applied to develop new core technology. Almost half of butterfly valve patents since 1985 have related to sealed issues, in order to improve the integral leakage index. Such as, a main trend of the technology evolution for the main structure of the valves is that the valve shaft and the valve disc in early valve are connected immovably (such as Chinese patent No. GG86208284). Later, they are connected jointly by one or more joints (such as Chinese patent No. ZL96241794.7 and No. 87208019U). Now, they are connected flexibly in some valves (such as Chinese patent application No. 94111763.4, the valve disc and valve shaft connected by a spring mechanism). So, evolution line of 'increased dynamics', which matched to this trend, was selected to find the technology opportunities in relation to the seal technology in the evolution of the butterfly valve. InventionTool 3.0 (China) is applied in this step.

Figure 5 shows the evolution line of increased dynamics:

stiff system $\rightarrow$ a joint $\rightarrow$ many joints $\rightarrow$ elastic $\rightarrow$ molecular (liquid, gas) $\rightarrow$ field

The current state for evolution of the main structures of the valve is in the "elastic", which matches the flexibility structure in some valves, and the states of "molecular" and "field" are potential states. The distance between the "field" state and the "elastic" state in the line exists the 'technology opportunities'. 

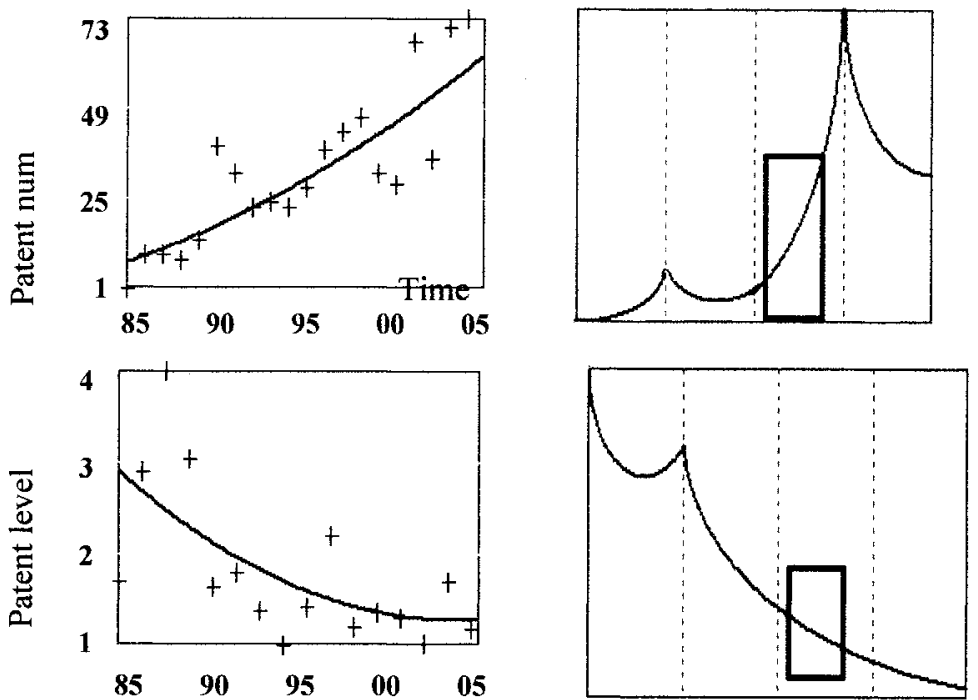

Time
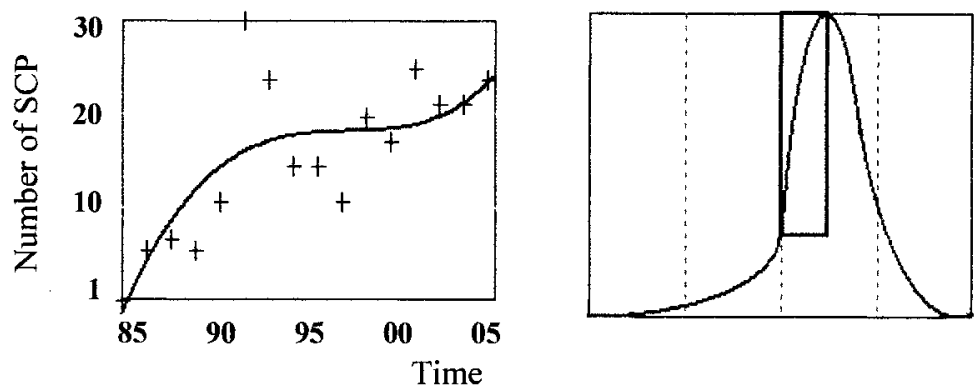

Fig. 4. The Product Patent Attribute Curves of butterfly valve versus classical curves

\section{Step 5: Generate strategic new ideas}

The key technique of the valve is to compensate for the wear of the seal ring and the valve disc end in order to reduce leakage. The action of the compensation is to make the valve disc to move forward in a small displacement after the rotation of the valve disc when closing the valve, or to make the valve disc to move backward in a small displacement before the rotation of the valve disc when opening the valve.

The application of the compressibility of oil or gas can implement the compensation function from the first potential state "molecular", the second potential state is also applicable. For example, a permanent magnet can implement the function of the compensation. So, some new ideas are generated:

Innovative ideas: Apply compressibility of liquid or gas, or a permanent magnet to implement the function of the seal ring compensation.

Step 6: Evaluate new ideas 
These innovative ideas should be possible because there are successful applications in industry or life. And they are generated from the 'technology opportunity' that other successful industries have already made, so the uncertainty of the new ideas is reduced. The engineers of the butterfly valve industry should also make evaluation for the ideas and implement them by the conditions of management, design and manufacture capacity of their firm, they should make an adaptive and specific design for the existing products that need to be improved. Accordingly, the pace of the products innovation is accelerated.

\section{Conclusions}

To generate innovative ideas for product development is a key step in the technical innovation process. CAI systems based on TRIZ are applicable to support this process. An integrated method is applied to assess the maturity of a technical system, and the result of TMM can guide the designer to decide between the optimization and innovation of the core technology. Then patterns and lines of technology evolution can be applied to find some technology opportunities of products and to forecast future technological R\&D plans.

A general process for technology innovation of product supported by CAI system is developed, with the aim of making the technology innovation more easily and practical. Technology innovation of the seal structure of butterfly valve is carried out and demonstrates the process step by step. The other patterns and lines of evolution can be applied to generate additional new ideas to provide a picture of the overall possible technological developments in butterfly valve technology, and to provide the decision-makers a striving direction for the new engineering products and technological innovation.

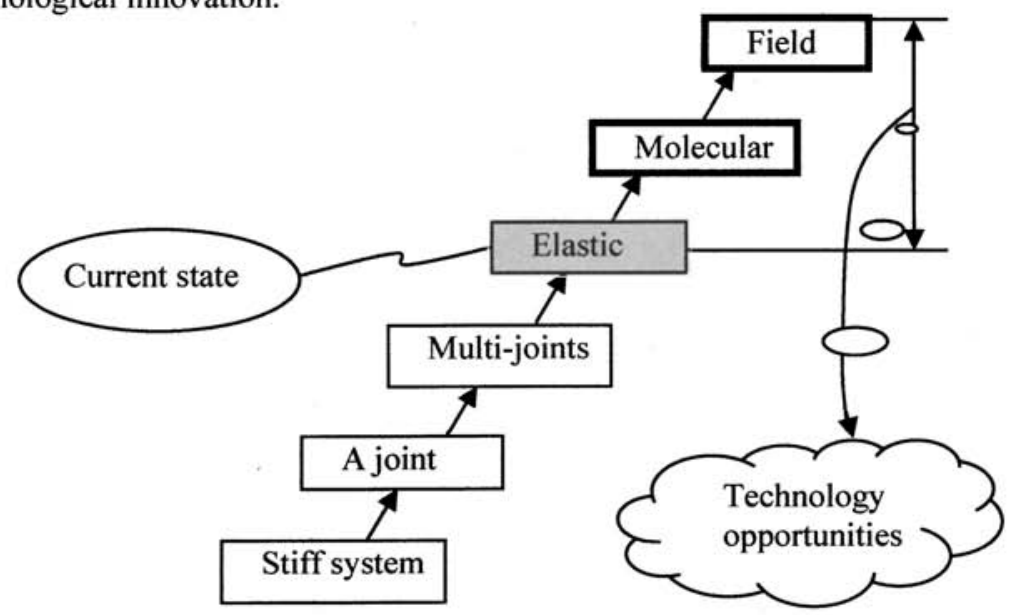

Fig. 5. 'Increasing the degree of system dynamics' evolution line. 


\section{Acknowledgments}

This research is supported by Natural Science Foundation of China under Grant Numbers 50675059, Chinese national 863 planning project under Grant Number 2006AA04Z109 and Education Office of Hebei Province of China under Grant Number 20062015. Authors are grateful to their fund for this work.

\section{References}

1. Severine Gahide, Application of TRIZ to Technology Forecasting Case Study: Yarn Spinning Technology, TRIZ Journal, July, 2000.

2. Altshuller G.S., Creativity As An Exact Science: The Theory of the Solution of Inventive Problems, The United Kingdom: Gordon and Breach Science Publishers Inc, London, 1984, pp. 205-216.

3. Tan Runhua, Innovation Design-TRIZ: the Theory of Inventive Problem Solving, China Machine PressBeijing, 2002, pp. 58-60. (in Chinese).

4. Michael S. Slocum, Technology Maturity Using S-curve Descriptors, TRIZ Journal, April, 1999.

5. Fey V R, Rivin E I., Guided Technology Evolution, http: //www.trizgroup.com/ article2.html, July, 2001.

6. Zhang Huangao, Tan Runhua, and Zhao Wenyan, The technology maturity of product and its mapping. ASME Proceedings of IMECE'04, IMECE2004-59260. Anaheim, California, USA, 2004.

7. Darrell Mann, Using S-Curves and Trends of Evolution in R\&D Strategy Planning, TRIZ Journal, July, 1999.

8. Alla Zusman, Boris Zlotin, Gafur Zainiev, An Application of Directed Evolution, http://www.Ideation-triz.com, June, 2001.

9. Tan Runhua, A Macro Process Model for Product Innovation Using TRIZ, the eighth world conference on integrated design and process technology, Beijing, China, 2005. 